\title{
Change Management as A Road Map for Safety Management System Implementation in Aviation Operations: Focusing on Risk Management and Operational Effectiveness
}

\author{
Prof. Dr. Triant FLOURIS \\ Dean, School of Aviation Sciences; \\ Professor, Aviation Management \\ School of Aviation Sciences, Daniel Webster College, \\ Nashua, NH 03063-1300, USA \\ flouris@dwc.edu \\ Dr. Ayse KUCUK YILMAZ \\ Assistant Professor, School of Civil Aviation; \\ Anadolu University, \\ 26470 Eskisehir, Turkey \\ akucukyilmaz@gmail.com
}

\begin{abstract}
This paper focuses on providing a theoretical answer to the question, how can an air carrier effectively implement a Safety Management System (SMS) in its operations? The core assumptions of this study are that (i) the value of a well-structured SMS in enhancing air carrier operational safety is axiomatic; (ii) Risk management is an integral part of safety management systems in aviation to provide effective aviation operations. We present a road map for change management, risk management and their utility and application in transforming a non-SMS compliant system to an SMS one using change management principles.
\end{abstract}

Keywords: Aviation Operations, Airline Management, Change Management, Risk, Risk Management, Safety Systems. 


\section{Introduction}

Safety is a non-negotiable attribute in the aviation industry. It is the cornerstone of any aviation operation and expected by customers, governments, and the public in general. Civil Aviation Authorities' primary goal is to safeguard, proactively, the safety of aviation operations. Commonly perceived as lack of accidents ${ }^{1}$ or incidents, aviation safety is primarily achieved by an organization through compliance with prescribed standards. Airlines are subject to strict regulatory oversight from their national authorities, which prevents them from deviating from safe operating standards. Furthermore, airlines and their suppliers are constantly audited by regulatory agencies, manufacturers, and other airlines, often following international standards such as IATA's Operational Safety Audit Program (International Air Transport Association).

Likewise, regulatory agencies themselves are overseen by ICAO's Universal Safety Oversight Program (USOAP). As a result of technological developments (notably Traffic Collision Avoidance Systems and Ground Proximity Warning Systems) as well as standardization initiatives, such as ICAO's Standards and Recommended Practices (SARPS) and other regulatory oversight, the airline industry has achieved considerable safety improvements since the 1960s. During these last decades, international aviation has witnessed an improvement in the rate of civil aviation accidents (Statistical Summary of Commercial Jet Airplane Accidents Worldwide Operations 1959 - 2004, Boeing Commercial Airplanes, May 2005).

Despite this, operators are still susceptible to error, which is not always preventable through regulatory oversight. Air carriers are still responsible for following safe operational practices to prevent accidents or incidents and are mandated by their national authorities to monitor their internal processes constantly to ensure that deviations are adequately addressed. For instance, complying with prescribed maintenance standards could, theoretically, maintain the probability of an accident due to mechanical failure at 1E-9 (1 event per 100 million opportunities). This is the acceptable level of safety risk for an individual catastrophic failure, as defined by national authorities during aircraft certification.

Air carriers are required to manage their operations adequately to ensure that their service, transporting passengers or cargo, is delivered in an efficient manner in order to satisfy stakeholder expectations. ${ }^{2}$ An airline can be perceived as an intricate network of departments, employees, contractors, and regulators interacting with each other. To conduct a safe operation, an airline's management needs to understand the complexities associated with its

\footnotetext{
1 ICAO (International Civil Aviation Organization) defines an airplane accident as the following: “an occurrence associated with the operation of an airplane that takes place between the time any person boards the airplane with the intention of flight and such time as all such persons have disembarked, and in which 1) the airplane sustains substantial damage; 2) death or serious injury results from being in or upon the airplane, direct contact with the airplane or anything attached thereto, or direct exposure to jet blast."

2 Stakeholders are the general public in addition to regulatory authorities.
} 
operations and develop, implement, and monitor control systems that will ensure compliance with safety standards. Moreover, the management of safety requires the organization to manage hazards particular to its operations proactively. Safety management has been recognized as a key aspect of an airline's operation and is now a regulatory requirement in many countries around the world. It is now recognized that the implementation of a Safety Management System (SMS) ${ }^{3}$ is a contributor to further reductions in aircraft accidents and incidents.

Is an SMS a panacea or just another buzzword that will be replaced with something new in a few years? How can an operator effectively implement an effective SMS in its operations? In this paper, we consider the value of a well-structured SMS in enhancing operational safety as an axiom and consider SMSs as nothing new. SMSs are deeply rooted in organizational behavior theory, and we propose that aviation operators, through the use of change management, can successfully transform existing systems into SMS compliant systems. Therefore, our argument focuses on how change management can be used as an effective technique in implementing a safety management system in an operator that is used to a non-SMS type system of regulatory compliance. Thus, we describe what change management is and how it can be utilized in an SMS transformation. By extension, though not a focus of this theoretical paper, our argument presupposes that an adequate management of safety is an indicator of the overall performance of an organization and, as such, a quality to be desired.

Airlines, employees and regulators all proceed with different concepts of risk, based on the perceptions of their people, their experience, public pressure and any number of other "environmental" factors. If differing risk strategies and views exist, then there are inevitable disagreements, any of which drag the operating process down. If the three Actors can come to agreement on the ideas of risk, then the operating process can go smoothly and efficiently (SMS Project Team of The Air Line Pilots Association, International, 2006). The objective of an SMS is to provide a structured management system to control risk in operations. Effective safety management must be based on characteristics of an operator's processes that affect safety (Federal Aviation Administration, 2006)

First, we briefly discuss organizational culture, define what an SMS is, and then proceed to define change management and propose ways the concept applies to the implementation of an SMS in an airline environment. In our discussion of organizational culture we propose that the stronger the culture in an organization, the more effectively the organization addresses change. By extension, the more effectively the organization addresses change, the more successful it can be in implementing a new SMS in its operations and the less it will need the deployment of radical change management techniques.

\footnotetext{
${ }^{3}$ For national authorities, a draft SMS manual has been released by ICAO (Doc 9859).
} 


\section{Review of Literature on Culture}

Definitions of organizational culture vary and typically depend on the academic discipline from which they originate. Business schools have the tendency to define organizational culture as a phenomenon that can be managed. Sociologists and anthropologists stress the uniqueness of individual organizations. Organizational psychologists with an empirical background believe that organizational culture can be broken down in its component parts and then studied part by part.

This paper defines organizational culture as the values, beliefs, assumptions, rituals, symbols, and behavior that define an organized group, especially in relationship to other organized groups and follows closely the business school definition. The visible part of organizational culture consists of observable behaviors and recognizable manifestations, for instance members' uniforms, symbols and logos, organizational routines and rituals, and printed documents. The deep layer of culture consists of the values, beliefs, and the subconscious assumptions that provide the logic, which guides the members' behaviors.

The management of safety in the aerospace industry has been linked to organizational culture. In fact, the proactive management of safety, including SMS initiatives, depends on the establishment of a hazard reporting culture (Reason, 1998). The important aspect of organizational culture vis-à-vis aviation safety outcomes is the underlying or deep culture. The visible aspect of culture is only procedural and is based on an organizational symbology. For instance, an employee, who is dissatisfied with his organization and not performing his duties to high standards, will still wear the company uniform to work. Therefore, in this scenario, the values, beliefs, and subconscious assumptions of the employee, vis-à-vis his organization, are sub-optimal and yet his appearance will appear normal.

The cultural strength of an organization has been defined by researchers in organizational management, sociology, and anthropology in a variety of ways. It has been defined as coherence (Deal and Kennedy, 1982), homogeneity (Ouchi and Price, 1978), stability and intensity (Schein, 1992), congruence (Schall, 1983), and internalized control (DiTomaso, 1987). Cultural strength relates to whom and how many accept the dominant values, how strongly these values are held, and how long the values have been dominant (Gordon \& DiTomaso, 1992). The underlying concept to cultural strength is the way in which employees accept these values, which is to say that employees must substantively believe in their organizational culture in order for the culture to be successful.

To believe in one's company's organizational culture substantively, an employee must be convinced of the superiority of this culture, and this culture must conform to his personality and national culture. However, this is complicated for organizations that exist in multicultural states $^{4}$ and companies that rely on expatriate personnel, thereby bringing a multitude of

\footnotetext{
${ }^{4}$ This is really not a major problem because in culturally diverse states the relevance of a national culture is evident, going beyond cultural or ethnic diversity and unifying people under one national framework. The United States is a good example of such a case.
} 
people from diametrically different cultures, ethnicities, and nations under one organizational rubric. Does cultural strength actually have an impact on organizational performance? Using an operationalization of cultural strength, ${ }^{5}$ two longitudinal studies have shown that a strong culture is predictive of organizational performance as measured by short-term profits and growth in assets (Denison, 1990; Gordon and DiTomaso, 1992).

\subsection{Defining SMS}

Effective safety management emphasizes the importance of managing safety in a systematic, proactive and explicit manner. Systematic means that safety management activities are conducted in accordance to a predetermined and well-documented plan and applied in a consistent manner throughout the organization. The existence of an integrated and strong company culture is an essential enabler in achieving this consistency. A strong and uniform company culture comes together with high morale amongst employees and good interdepartmental links and communication systems throughout an organization. Proactivity means adopting an approach which emphasizes prevention through the identification of hazards and the introduction of risk mitigation measures before the risk-bearing event occurs and adversely affects safety performance. If this type of an organizational practice already exists in a company culture that emphasizes incident and accident prevention, rather than a reactive culture that focuses on solving problems after they occur, then an SMS becomes easier to implement. Finally, what is explicit in an SMS system is the fact that all safety management activities should be well-documented and in a clear manner. In addition, they should be visible both to inter as well as intra organizational stakeholders and be performed independently from other management activities. Safety becomes a uniform focus for the organization, rather than an afterthought, and responsibility for its management is delegated to a specific organizational unit whereas, at the same time, is pervasive in the practices of the organization as a whole.

Essential practices that are associated with safety management include the following: hazard identification and the closing of gaps in defending an existing system. This practice is related to the principle of proactive management in that quality assurance is a dynamic process that is achieved through the use of some sort of an ever evolving and improving total quality management system. Additionally, effective safety management is multi-disciplinary; it involves several departments within the organization. More specifically, even though the organization's safety department has core competency vis-à-vis safety and in promulgating a safety culture throughout the organization, the know-how of technical experts in a variety of other areas is equally valuable. These experts are involved in the day-to-day practice of safety and should be allowed to offer input in the proactive solution of potential hazards. This approach requires that an appropriate and systematic application of a variety of techniques and activities are utilized in an array of situations, and this is done in a way that fits the

\footnotetext{
${ }^{5}$ Cultural strength was measured based on the consistency rather than the content of employee responses to survey items about organizational culture.
} 
specific problem. Thus, for example, if the problem is one of a human resource process, the appropriate expert with a disciplinary competence best suited to take a lead in identifying and solving a specific gap is called upon to contribute to the solution.

Effective SMSs are built across three defining cornerstone characteristics. First, a comprehensive corporate approach to safety has to be assured, which sets the tone for the management of safety, builds upon the safety culture of the organization, embraces the organization's safety policies, objectives and goals, and ensures that senior management is fully committed to safety. Secondly, effective organizational tools to deliver safety standards must exist. These tools are needed to deliver the necessary activities and processes to advance safety. They are also important in arranging organizational matters in order to fulfill safety policies, objectives and goals. They establish standards and allocate resources as well as focus on hazards and their potential effects on safety-critical activities. Finally, a formal system for safety oversight is needed to confirm the organization's continuing fulfillment of its corporate safety policy, objectives, goals and standards. It is important, along the parameters of this discussion, that it is understood that the scope of the SMS be appropriate to the size and complexity of the operation. Therefore, a one-size and scope fits all approach is not commensurable with an effective SMS.

\subsection{Moving to SMS as a Restructuring Exercise: What Is Change Management?}

Restructuring implies change. So how is change at an airline that is moving to an SMS system managed? This study focuses on the theory of change as it relates to any organization. In this effort, we present definitions of change, models of the organization, an outline of the change process, and a diagnostic model for identifying where change should take place. Another study will have to be conducted where the theory and concepts of change are applied to a specific airline case study, which includes recommendations for managing the change process.

All organizations by definition take inputs from the external environment, transform those inputs internally through their existing organizational processes, and then produce outputs, which again are directed at the external environment. As such, organizations can be considered open systems, and thus are susceptible to developments in their business environment since they are part of that environment. When an organization's internal structure and functions are organized such that they can exploit the external environment to their advantage, then an organization can be considered to be operating effectively. However, the external environment is in most cases more dynamic and fluid than that of a typical organization. Changes in the external environment can happen so quickly that almost overnight organizations can find themselves unable to deal effectively with the situation. If an organization wishes to survive, it too must change. The ability of managers to guide and influence the outcome of changes is Change Management. These abovementioned statements are particularly true in the case of Safety Management System 
implementation by air carriers. The inability of an airline to transfer itself effectively in a Safety Management System, especially if it is a new regulatory standard or widely acceptable industry practice, will have a negative impact on its efficiency.

\subsection{Types of Change}

As mentioned earlier, change is initiated in the external environment, and this requires firms to change in order to remain effective. In the case of an SMS, its acceptance as a good global safety practice through ICAO and IATA has been instrumental in its broader acceptance. The external environment with respect to any firm or collection of firms in a similar industry can be characterized by the amount of change that is occurring over time in the environment. Broadly speaking the external environment can be considered to be in either equilibrium (in the airline industry consider the era of regulation), which implies only small incremental changes in the way a firm operates. On the other hand, the external environment can be characterized by a period of disequilibrium. Disequilibrium is often triggered by a destabilizing event, or set of events, that change the basic dynamics or relationships in a particular industry. The destabilizing event can be triggered from one of the industry participants (as it attempts to gain a competitive advantage) or it can come from outside the industry (fears of terrorism, for example) (Haynes, 2002). The events of September $11^{\text {th }} 2001$ provide us with an example of an external destabilizing agent for the airline industry.

Thus, two types of change can be considered to affect the organization: incremental and discontinuous. Incremental change is a type of change associated with those periods when the industry in equilibrium and the focus of change for the organization is to do things better, through continuous change, adaptation and modification. ${ }^{6}$ In contemporary language this type of change is often referred to as continuous improvement.

On the other hand, discontinuous change is change that occurs in periods of disequilibrium. ${ }^{7}$ This type of change is sometimes called transformational change, as the organization that undergoes such change must completely break with its past and find new ways to operate. In addition to new operational methods, an organization facing transformational change must also create and define a completely new set of strategies, since previous core competencies may have been undermined due to changes in the external environment. In essence this type of change requires the organizations to do things differently rather than doing things better. It may even mean doing completely different things ${ }^{8}$ as can be the case with an SMS.

In addition to the concept of incremental and discontinuous change, two other categories of change can be defined, both of which incorporate the element of time. First, there is anticipatory change, which is initiated by a firm without a clear external demand. This type

\footnotetext{
${ }^{6}$ Ibid.

7 Ibid.

${ }^{8}$ Ibid.
} 
of change might be undertaken by a firm in order to gain a competitive advantage in the market place or to prepare for a likely future event. Anticipatory change happens before an event in the external environment. Second, there is reactive change, which is a firm's response to a clear and present danger that already exists at present in the external environment. Movement towards the design and implementation of a safety management system for an airline can be attributable to either anticipatory or reactive change and depends on how the airline in question brings it about. For example, if an SMS is mandated by a national regulatory authority as the new regulatory standard, an airline that has not taken steps to change its existing system to an SMS type system must react to the new regulatory standard. On the other hand, an airline that starts using an SMS system in anticipation of regulatory change or in order to self-generate more efficiency in its safety management operations is said to engage in anticipatory change. Efficiency here is defined as the internal ability to do more with less or the same with less while maintaining or improving safety outcomes.

Combining the concepts of equilibrium together with that of time, a change matrix for organizations can be constructed as follows (Figure 1):

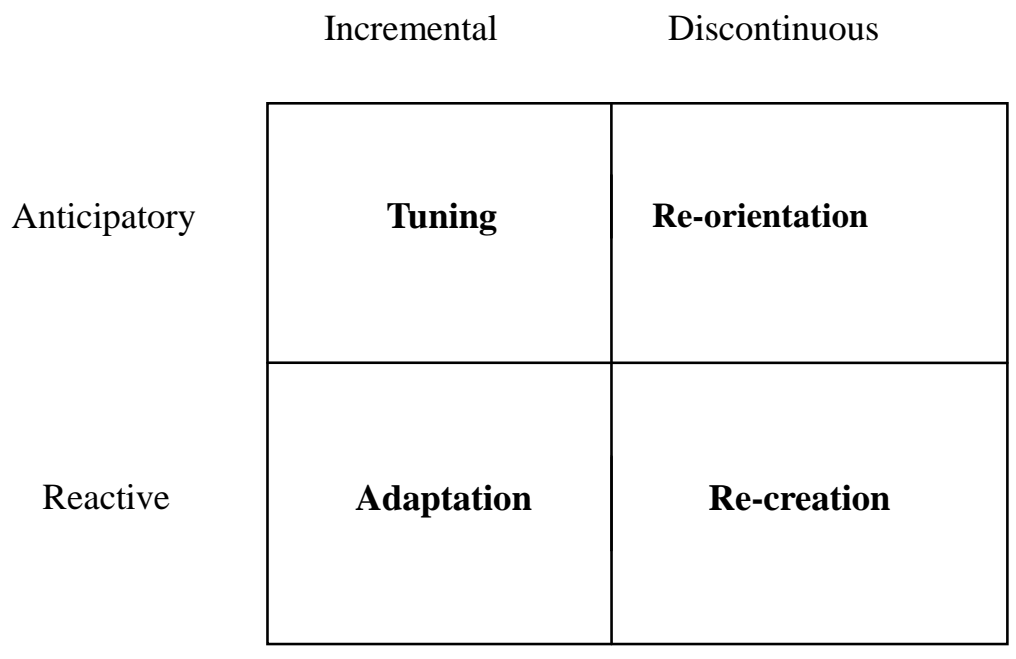

Figure 1: Types of Organizational Change (Hayne, 2002)

Defining a change matrix as above is a useful tool that enables us to make preliminary diagnoses of the type of change facing an organization. As will be illustrated later, knowing the type of change facing an organization will greatly assist directing resources and time to the appropriate places within the organization.

Tuning is simply a change that is undertaken when there is no immediate requirement for change. Essentially this type of change is associated with fine tuning an existing strategy. Adaptation is similar to tuning but is undertaken due to the presence of some external factor. 


\section{Macrothink

This type of change essentially means "doing things better." Re-orientation is a wholesale change undertaken by an organization in anticipation of some future event. The aim of this type of change is to ensure that the organization remains aligned with the external environment. Finally, re-creation is a fundamental realignment of the firm due to events currently taking place in the environment. Both re-orientation and re-creation require the organization to dramatically change all of its elements. This includes a change in strategies (corporate and business), and thus implies old core competencies may need to be abandoned in order that new, more effective competencies be developed. SMS implementation in a non-SMS environment falls under any of the abovementioned categories. The magnitude and type of change that are required depend on the degree to which an air carrier's existing system is already aligned with specific SMS requirements. By this we mean how close the air carrier's existing system is to an SMS compliant system from an organizational or safety culture strength perspective.

\subsection{Why Do Organizations Need to Change?}

Thus far, basic definitions of change have been described and defined. What has not been discussed, however, is why organizations need to change. From the preceding discussion it is probably obvious to the reader that an organization's ability to navigate change is directly related to its organizational effectiveness and performance.

Figure 2 describes a typical decline process that occurs when an organization fails to give proper regard to changes in its external environment. In the first stage (Blinded), organizations are unable to recognize internal and external changes that may affect the long term survival of the organization. In the second stage (Inaction), organizations fail to respond to a need for change despite signs of worsening performance. In the third stage (Faulty Action), the organization takes actions but these actions are inappropriate. In the fourth stage (Crisis), after failing to deal with the problems facing it, the organization finds itself in crisis. Finally, failure to respond to the crisis results in the eventual death or dissolution of the organization. While Figure 2 makes obvious the process of decline, another point is worth mentioning. It seems intuitive that an organization in decline should respond to the change it faces, which has put it in a position of vulnerability, in an appropriate manner rather than over or under react. For example, adequate identification of threats in the external environment may prompt the organization to make small incremental changes (tuning), rather than large scale transformational changes (recreation). 


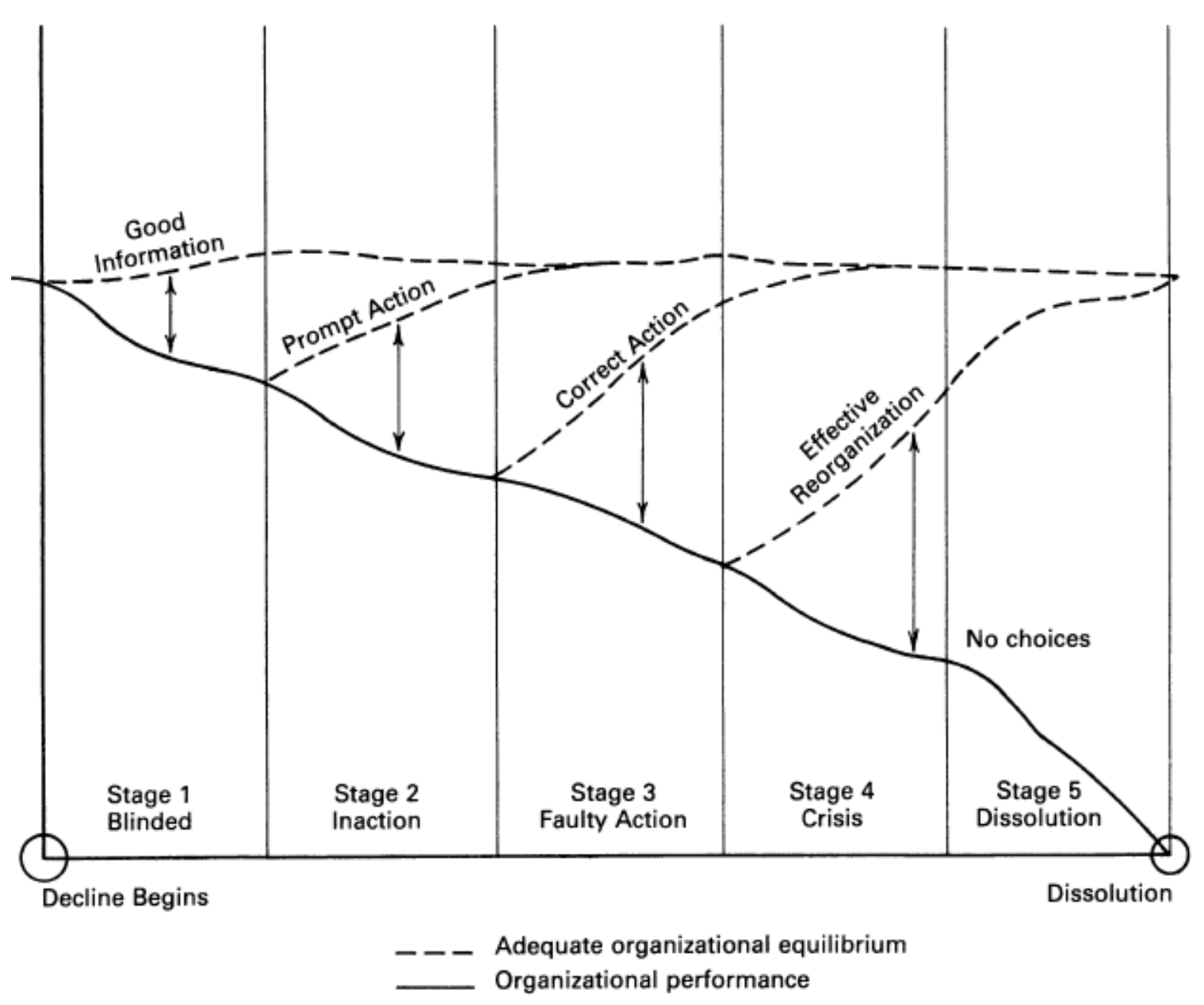

Figure 2: Widening Performance Gap (Hall, 2002)

\subsection{The Change Process}

The change process is a dynamic and fluid one. Generally, change can be categorized into three basic stages. The first stage is the unfreezing process where the organization leaves or alters its existing levels of behavior. The second stage involves moving to a new behavioral level. The third stage is refreezing at this new level. By refreezing what is meant is that new behaviors have supplanted old ones resulting in a new set of behaviors for the organization. For example, the management of safety requires the organization to manage hazards particular to its operations proactively. If proactive management does exist, an airline that wants to move in this direction can "unfreeze" current processes which prevent it from doing so (for example, unwillingness to speak up if a mistake is detected for fear of management or colleague retaliation), then, through a training program, re-align the behavioral pattern of its employees (moving to a new behavioral level), and finally refreeze 
the organizational process once adequate evidence is presented that behavior modification has taken place (refreeze). With this in mind Figure 3 presents a process view of change.

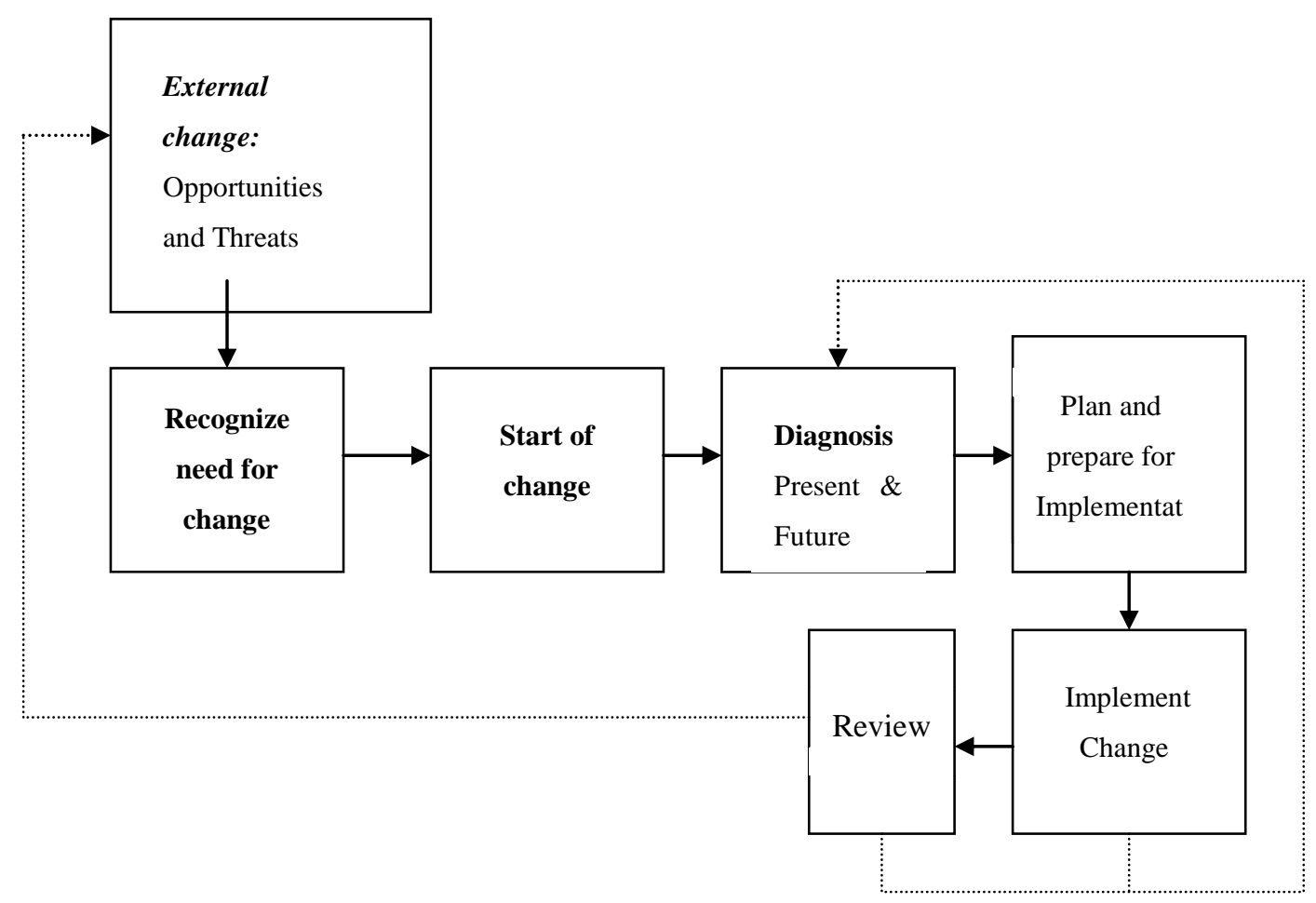

Figure 3: The Change Process (Hayes, 2002)

Note that this model reflects on and extends the three stage model for change presented earlier. The first of the three steps of the process view essentially represent the unfreezing stage as the organization first observes changes in its external environment, translates this perception into a need, and thus begins the change process. The diagnosis and implementation planning represent the movement from the previous state to the new state. The implementation and review stages of the process model represent the beginning of refreezing where new behaviours are absorbed into the organization. Finally, we should note that this is a continuous process, and that refreezing does not mean that the organization is locked into a new behavior, but rather that new modes of operation have been learnt and integrated into the organization. It is like a dialectic process, where continuous change and adaptation are not just necessary but inevitable if the organization is to survive.

The most critical steps of the change process are the diagnosis stage and the transition to the implementation phase. The diagnosis phase is important as the organization must determine where organizational performance is being adversely affected and needs to be changed. The implementation plan then sets out to correct or modify the defects noted in the diagnosis and represents a crucial step towards re-establishing organizational effectiveness. 


\subsection{Diagnosing Where to Change}

There are several models available in order to diagnose change. The Burke-Litwin model (Figure 4) is very useful as it describes twelve interrelated elements of an organization. At the top of the diagram is the external environment, and this represents the inputs for an organization. At the bottom of the diagram is the performance of the individual and organization and as such represents the output. The area in between represents how an organization turns inputs into outputs and, thus, represents the key activities and elements of an organization. Furthermore, the model is organized in a vertical fashion to indicate the relative impact that one element has over another element in the organization. For example, the organizational culture will affect both the work unit climate and individual needs and values. While the work unit climate can affect the organizational culture, this model posits that organizational culture has a much greater weight or force on the work unit climate than vice-versa.

What makes this model most interesting for diagnosing change, however, is the fact that it inherently distinguishes between transformational change and transactional change. Earlier in the paper, change was broadly categorized into either Incremental or Discontinuous. An organization faced with the former need only to modify itself in order to do things better (transactional change), while the latter required the organization to drastically remodel itself (transformational change).

As transactional change is focused on minor "tuning," change efforts need to be directed at the structures, management practices, and systems, which affect the work climate unit that in turn affects motivation and performance of both the individual and organization (Hayes, 2002).

On the other hand, it is clear that when an organization is confronted with transformational change efforts for change must be directed higher up in the organization. In other words, this type of radical change calls for a reworking of the organization's mission and strategy, its leadership and its organizational culture. As the model implies, changes at this relatively high level will be transmitted through the lower levels and, thus, may well cause incremental change to occur as well.

\subsection{Improving to SMS as a Supporting and Integrating Exercise: Risk Management based Approach?}

The efficient and effective management of any aviation organization, regardless of the nature of its functions or its size, requires the management of basic and traditional business processes: financing, budgeting, communicating, allocating resources and so forth. In recent years, managing safety has been added to the list of basic and traditional business processes. Managing safety risk should now be as much a part of running an aviation organization as 
managing any of the traditional business processes. The term safety risk management conveys the notion that the management of safety is a business process that must be considered at the same level and along the same lines as any other business process (Vince et all, 2008:1)

The aviation industry is both complex and unique. The demands on employees are great and, in many cases, the requirements are not accurately communicated to upper management. Passengers and regulators both demand an increased level of safety. This is emphasized in recent legislative requirements for airlines to implement a systematic approach to safety management or SMS. The core requirement for an SMS is an effective method of identifying and controlling risk. The concept is described by ICAO as "The risks and costs in commercial aviation necessitate a rational process for decision-making. Daily, operators and managers make decisions in real time, weighing the probability and severity of any adverse consequences implied by the risk against the expected gain of taking the risk. This process is known as risk management." (SCSI, 2009)

Risk Management is a systems-based approach that focuses on the identification of hazards involved in each aspect of the operation, whether it involves aircraft flight operations, cockpit procedures, aircraft maintenance, turn-around, ticketing, scheduling, or baggage handling. As an integral and required part of a Safety Management System, Operational Risk Management formalizes this approach by implementing a logic-driven process to analyze the degree of risk associated with identified hazards, recommending Risk-based solutions, and monitoring the effectiveness of these solutions (SCSI, 2009).

Following Figure 4 shows how the safety risk management and safety assurance processes are integrated in the SMS. The safety risk management process provides for initial identification of hazards and assessment of risk. Organizational risk controls are developed and, once they are determined to be capable of bringing the risk to an acceptable level, they are employed operationally. The safety assurance function takes over at this point to ensure that the risk controls are being practiced and they continue to achieve their intended objectives. This system also provides for assessment of the need for new controls because of changes in the operational environment (Federal Aviation Administration, 2006). 


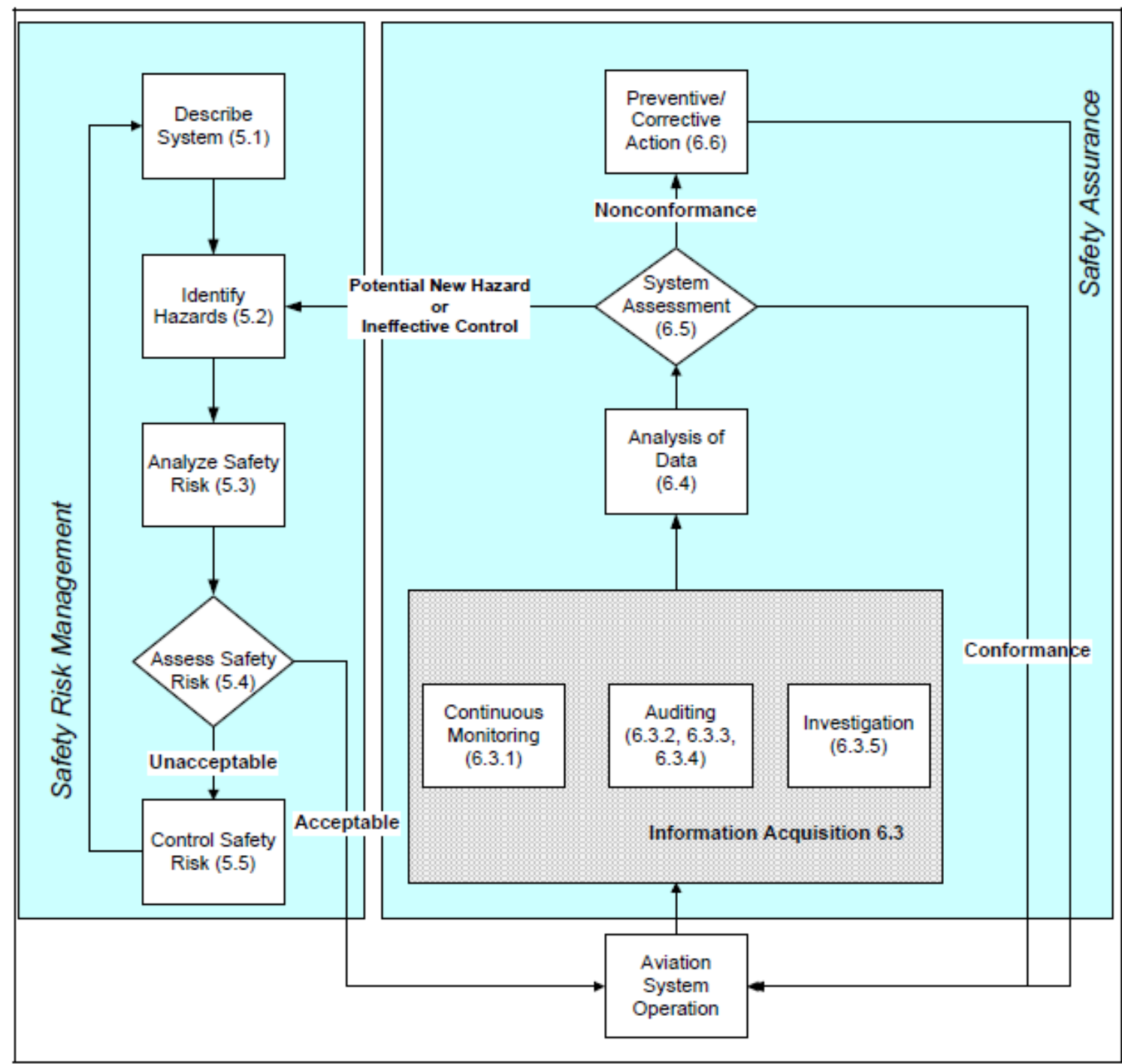

Figure 4: Safety Risk Management and Safety Assurance Processes (FAA, 2006) (The numbers in the process blocks shown in Figure 3 refer to clause numbers in the SMS standard in Appendix 1).

\section{Conclusion}

In order to conduct efficient and effective aviation operations and to be aware of the safety based risk factors, operators should have risk and change management based safety systems. Operators in the aviation sector must improve and maintain a high level of safety by proactive risk management and holistic change management integration into safety management systems in order to minimize accidents in airline operations.

This theoretical study shows that SMS is a concept that has existed in academic literature for quite some time. Furthermore, its application in the aviation industry in transforming existing safety compliance systems is tied to the application of change management and risk 
management principles to administer the SMS procedures. We assume that the existence of strong organizational cultures would make change management and risk management in establishing SMS systems entertain higher likelihoods of success. The most critical steps of the change process for an airline that moves from a conventional system of safety compliance to an SMS type system are the diagnosis stage and the transition to the implementation phase. The diagnosis phase is important as the organization must determine where organizational performance is being adversely affected and needs to be changed. The implementation plan then sets out to correct or modify the defects noted in the diagnosis and represents a crucial step towards re-establishing organizational effectiveness. Empirical research through case studies of SMS implementation will be necessary to test the hypotheses presented in this paper.

\section{References}

Federal Aviation Administration, (2006), Introduction to Safety Management systems fora ir Operators, Advisory Circular, AFS-800, AC no: 120-92, Par 2, p.4.

Galotti, Vince; Rao, Arun; Maurin, Daniel. (2008), Implementation of Safety Management Systems (SMS) in States Article, Sarps and Guidance Material, Library, The United Kingdom Flight Safety Committee, p.1.

Hall, R. (2002), Organizations: Structures, Processes, and Outcomes, $8^{\text {th }}$ edition, Prentice-Hall, New Jersey.

Haynes, J. (2002), The Theory and Practice of Change Management, Palgrave, Hampshire.

International Air Transport Association, (2009) www.iata.org/

SCSI ( 2009 ). Operational Risk Management, http://www.scsi-inc.com/orm.html.

SMS Project Team of The Air Line Pilots Association, International, (2006), Background and Fundamentals of the Safety Management System (SMS) for Aviation Operations, Second Edition, February, 2006, p.24.

Statistical Summary of Commercial Jet Airplane Accidents Worldwide Operations 1959 2004, Boeing Commercial Airplanes, May 2005, www.aermalignani.org/pdf/boeing.pdf For national authorities, a draft SMS manual has been released by ICAO (Doc 9859). 


\section{Appendix 1(FAA, 2006)}

\subsection{System and Task Analysis}

A) System and task descriptions shall be developed to the level of detail necessary to identify hazards.

B) System and task analyses should consider the following:

1) The system's interactions with other systems in the air transportation system (e.g. airports, air traffic control);

2) The system's functions for each area listed in paragraph $4.1 \mathrm{~A}$ );

3) Employee tasks required to accomplish the functions in 5.1 B) 2);

4) Required human factors considerations of the system (e.g. cognitive, ergonomic, environmental, occupational health and safety) for:

a) Operations, and

b) Maintenance;

5) Hardware components of the system;

6) Software components of the system;

7) related procedures that define guidance for the operation and use of the system;

8) Ambient environment;

9) Operational environment;

10) Maintenance environment;

11) Contracted and purchased products and services;

12) The interactions between items in Section

5.1.B., 2 - 10 above; and

13) Any assumptions made about:

a) The system,

b) System interactions, and

c) Existing safety risk controls.

\subsection{Identify Hazards}

A) Hazards shall be:

1) Identified for the entire scope of the system that is being evaluated as defined in the system description; and

2) Documented.

B) Hazard information shall be:

1) tracked, and

2) managed through the entire SRM process.

5.3. Analyze Safety Risk The safety risk analysis process shall include:

1) existing safety risk controls;
2) triggering mechanisms; and;

3) safety risk of reasonably likely outcomes from the existence of a hazard, to include estimation of the: a) likelihood; and b) severity.

\subsection{Assess Safety Risk}

A) Each hazard shall be assessed for its safety risk acceptability using the safety risk objectives described in Section 5D.

B) The organization shall define levels of management that can make safety risk acceptance decisions.

\subsection{Control Safety Risk}

A) Safety control/mitigation plans shall be defined for each hazard with unacceptable risk.

B) Safety risk controls shall be:

1) clearly described;

2) evaluated to ensure that the requirements have been met;

3) ready to be used in the operational environment for which they are intended; and

4) documented.

C) Substitute risk shall be evaluated in the creation of safety risk controls/mitigations.

\section{Safety Assurance and Internal Evaluation}

Figure 3 illustrates how Safety Assurance functions (described in Sections $6.2-6.6$ ) are linked to the SRM process (described in Section 5).

\subsection{General Requirements}

The organization shall monitor heir systems and operations to:

1) identify new hazards;

2) measure the effectiveness of safety risk controls; and

3) ensure compliance with regulatory requirements.

\subsection{System Description}

The safety assurance function shall be based upon a comprehensive system description as described in Section 5.1.

\subsection{Information Acquisition}

The organization shall collect the data necessary to demonstrate the effectiveness of the organization's:

1) Operational processes; and

2) the SMS. 


\subsubsection{Continuous Monitoring}

A) The organization shall monitor operational data (e.g., duty logs, crew reports, work cards, process sheets, or reports from the employee safety feedback system specified in Section 7.1.5 to:

1) assess conformity with safety risk controls (described in Section 5);

2) measure the effectiveness of safety risk controls (described in Section 5);

3) assess system performance; and

4) identify hazards.

B) The organization shall monitor products and services received from subcontractors.

6.3.2 Internal Audits by Operational Departments

A) Line management of operational departments shall ensure that regular internal audits of safety-related functions of the organization's operational processes (production system) are conducted. This obligation shall extend to any subcontractors that they may use to accomplish those functions.

B) Line management shall ensure that regular audits are conducted to:

1) determine conformity with safety risk controls; and

2) assess performance of safety risk controls.

C) Planning of the audit program shall take into account:

1) safety significance of the processes to be audited; and

2) the results of previous audits.

D) The audit program shall include:

1) definition of the audit:

a) criteria,

b) scope,

c) frequency, and

d) methods;

2) the processes used to select the auditors;

3) the requirement that individuals shall not audit their own work;

4) documented procedures, which include:

a) the responsibilities; and b) requirements for:

(1) planning audits,

(2) conducting audits,

(3) reporting results, and

(4) maintaining records; and

5) audits of contractors and vendors.

\subsubsection{Internal Evaluation}

A) The organization shall conduct internal evaluations of the operational processes and the SMS at planned intervals to determine that the SMS conforms to requirements.

B) Planning of the evaluation program shall take into account:

1) safety significance of processes to be audited; and

2) the results of previous audits.

C) The evaluation program shall include:

1) definition of the evaluation:

a) criteria;

b) scope;

c) frequency; and

d) methods;

2) the processes used to select the auditors;

3) the requirement that auditors shall not audit their own work;

4) documented procedures, which include:

a) the responsibilities, and

b) requirements for:

(1) planning audits,

(2) conducting audits,

(3) reporting results,

(4) and maintaining records; and

5) audits of contractors and vendors.

D) The program shall be under the direction of the management official described in Section 4.5.

E) The program shall include an evaluation of the program required described in Section 6.3.2.

F) The person or organization performing evaluations of operational departments must be functionally independent of the department being evaluated.

\subsubsection{External Auditing of the SMS}

A) The organization shall include the results of oversight organization audits in the analyses 
conducted as described in Section 6.4.

\subsubsection{Investigation}

A) The organization shall collect data on:

1) incidents, and

2) accidents.

B) The organization shall establish procedures to:

1) investigate accidents;

2) investigate incidents; and

3) investigate instances of potential regulatory non-compliance.

\subsubsection{Employee Reporting and Feedback System.}

A) The organization shall establish and maintain a confidential employee safety reporting and feedback system as in Section 7.1.5).

B) Employees shall be encouraged to use the safety reporting and feedback system without reprisal as in Section 4.2 B) 5).

C) Data from the safety reporting and feedback system shall be monitored to identify emerging hazards.

D) Data collected in the safety reporting and feedback system shall be included in analyses described in Section 6.4.

\subsection{Analysis of Data}

A) The organization shall analyze data the data described in Section 6.3 to demonstrate the effectiveness of:

1) risk controls in the organization's operational processes, and

2) the SMS

B) Through data analysis, the organization shall evaluate where improvements can be made to the organization's:

1) operational processes, and

2) SMS.

\subsection{System Assessment}

A) The organization shall assess the performance of:

1) safety-related functions of operational processes against their requirements, and

2) the SMS against its requirements.

B) System assessments shall result in a finding of:

1) conformity with existing safety risk control(s)/ SMS requirement(s) (including regulatory requirements);

\subsubsection{Investigation}

A) The organization shall collect data on:

1) incidents, and

2) accidents.

B) The organization shall establish procedures to:

1) investigate accidents;

2) investigate incidents; and

3) investigate instances of potential regulatory non-compliance.

6.3.6 Employee Reporting and Feedback System.

A) The organization shall establish and maintain a confidential employee safety reporting and feedback system as in Section 7.1.5).

B) Employees shall be encouraged to use the safety reporting and feedback system without reprisal as in Section 4.2 B) 5).

C) Data from the safety reporting and feedback system shall be monitored to identify emerging hazards.

D) Data collected in the safety reporting and feedback system shall be included in analyses described in Section 6.4.

\subsection{Analysis of Data}

A) The organization shall analyze data the data described in Section 6.3 to demonstrate the effectiveness of:

1) risk controls in the organization's operational processes, and

2) the SMS.

B) Through data analysis, the organization shall evaluate where improvements can be made to the organization's:

1) operational processes, and

2) SMS.

\subsection{System Assessment}

A) The organization shall assess the performance of:

1) safety-related functions of operational processes against their requirements, and

2) the SMS against its requirements.

B) System assessments shall result in a finding of:

1) conformity with existing safety risk control(s)/ SMS requirement(s) (including regulatory 
requirements);

\subsection{Preventive/Corrective Action}

A) The organization shall develop, prioritize, and implement, as appropriate:

1) corrective actions for identified nonconformities with risk controls; and

2) preventive actions for identified potential nonconformities with risk controls actions.

B) Safety lessons learned shall be considered in the development of:

1) corrective actions; and
2) preventive actions.

C) The organization shall take necessary corrective action based on the findings of investigations.

D) The organization shall prioritize and implement corrective action(s) in a timely manner.

E) The organization shall prioritize and implement preventive action(s) in a timely manner.

F) Records shall be kept of the disposition and status of corrective and preventive actions per established record retention policy. 